\title{
Plasma generation in vibrationally nonequilibrium molecular gas flows
}

\author{
O. V. Achasov, N. A. Fomin, D. S. Ragozin, R. I. Soloukhin and S. A. Zhdanok \\ Heat and Mass Transfer Institute, Minsk 220728, USSR
}

(Reçu le 2 juin 1981, révisé le 15 septembre 1981, accepté le 12 octobre 1981)

\begin{abstract}
Résumé. - L'ionisation associative des molécules $\mathrm{N}_{2}$ et $\mathrm{CO}$ en refroidissement adiabatique après chauffage en tube à choc est étudié expérimentalement et théoriquement. On montre que dans un large intervalle de conditions, la contribution des méchanismes d'ionisation associative dépasse considérablement (de plusieurs ordres de grandeur) la contribution thermique.
\end{abstract}

\begin{abstract}
Experimental and theoretical studies are made of the associative molecular gas ionization phenomena in $\mathrm{N}_{2}$ and $\mathrm{CO}$ under the adiabatic expansion and cooling of a shock-heated gas. It is shown that for a wide range of conditions, the contribution of the associative ionization mechanism substantially exceeds (by several orders of magnitude) the thermal one.
\end{abstract}

1. Introduction. - Ionization processes in molecular gases are of great importance in the developments of high-power gas lasers as well as in plasma chemistry, high-temperature gasdynamics and atmosphere physics. Ionization due to electron impact and photoionization are widely met in experiments with high pressure glow discharges in molecular gases and are at present well understood [1]. It has been stated in [2] that an associative molecular gas ionization may occur as a result of the vibrationallyexcited molecule collisions according to a scheme :

$$
\begin{aligned}
& \mathrm{A}\left(v_{1}\right)+\mathrm{A}\left(v_{2}\right) \rightarrow(\mathrm{AA})^{+}+\mathrm{e} \quad\left(\text { or } \mathrm{A}+\mathrm{A}^{+}+\mathrm{e}\right) \\
& \text { if } \quad E\left(v_{1}\right)+E\left(v_{2}\right)>E_{\mathrm{i}}
\end{aligned}
$$$$
\text { if }
$$

where $\mathrm{A}(v)$ is the molecule in the $v$-th vibrational state with energy $E(v)$ and $E_{\mathrm{i}}$ is the ionization energy. $(\mathrm{AA})^{+}$and $\mathrm{A}^{+}$are the complex and ordinary ions of a molecule $\mathrm{A}$. The recombination reaction step, inverse to (1), contributes to the electron balance in the gas discharge plasma and is well known experimentally [3]. The investigation of the direct reaction (1) in the electric discharge involves considerable difficulties connected with a great variety of plasma chemical reactions which results in molecule ionization, and a contribution of this particular reaction is difficult to separate among a great number of possible ionization mechanisms in the experiments.

The associative ionization can be more correctly studied by measuring the molecular gas conductivity under high vibrational excitation conditions in the absence of any other sources of charged particles. These conditions can be easily implemented under the fast adiabatic expansion of molecular gases in a supersonic nozzle flow. In this case, since the vibrational-translational $(V-T)$ relaxation time strongly depends on gas temperature, the vibrational energy substantially exceeds the translational and rotational ones. As the test gas is significantly cooled due to the adiabatic expansion, the thermal ionization degree under these conditions is negligibly small, and the reaction (1) becomes the only possible electron source. The first experiments on the associative nitrogen ionization observations under the adiabatic nozzle expansion have been reported in [4]. The results obtained have confirmed the existence of the associative ionization mechanism based on the vibrationally excited molecules interaction and shown its high efficiency. The present study is devoted to a systematic investigation of the associative ionization reactions in expanding flows of $\mathrm{CO}$ and $\mathrm{N}_{2}$. The data obtained are compared with the corresponding available experimental data obtained under the gas discharge plasma conditions in $\mathrm{N}_{2}$ and $\mathrm{CO}$.

2. Experimental. - The schematic of the pulsed gasdynamic installation used for studying quasisteady vibrationally nonequilibrium gas flows was presented in $[5,6]$. The shock tube, $5 \times 5 \mathrm{~cm}^{2}$ in cross-section, was utilized to provide an adiabatic compression of a test gas up to pressures of 3 to $30 \mathrm{~atm}$ and temperatures in the range of 1 to $6 \times 10^{3} \mathrm{~K}$. The quasi-steady flow time developing in the shock 
tube end section, at the test gas $\left(\mathrm{N}_{2}, \mathrm{CO}\right)$ pressures of 0.03-0.1 atm. in the shock-tube expanding flow, was about 0.1 to $0.2 \mathrm{~ms}$ while that of a steady-flow was 0.5 to $1 \mathrm{~ms}$. The nozzle section was separated from the main shock-tube channel by a thin hatched copper diaphragm and was pre-evacuated, which ensured a rapid quasi-steady development and substantial increase in its working time [5]. The stagnation temperature and pressure of a shocked gas before expansion were determined with the use of the shock adiabatic relations at the given measured shock velocity.

The nozzle design and its parameters were similar to those utilized in the gasdynamic laser experiments [6]. The nozzle throat height was $0.55 \mathrm{~mm}$, the expansion area ratio $A / A^{*}$ was equal to 52 , and the output flow Mach number was equal to 6 . The sharpedge fast expansion Prandtl-Meyer nozzle, $5 \mathrm{~cm}$ in length, was connected with the constant cross section shock tube channel. Such a nozzle design can provide a fast decrease in the translational gas temperature in the nozzle throat area, at the uniform flow conditions, and hence it possesses the highest efficiency for the vibrational energy freezing process. The constant area shock channel walls were made of dielectric (textolite), in which the round $1 \mathrm{~cm}$ dia, copper, electrodes were installed (Fig. 1).
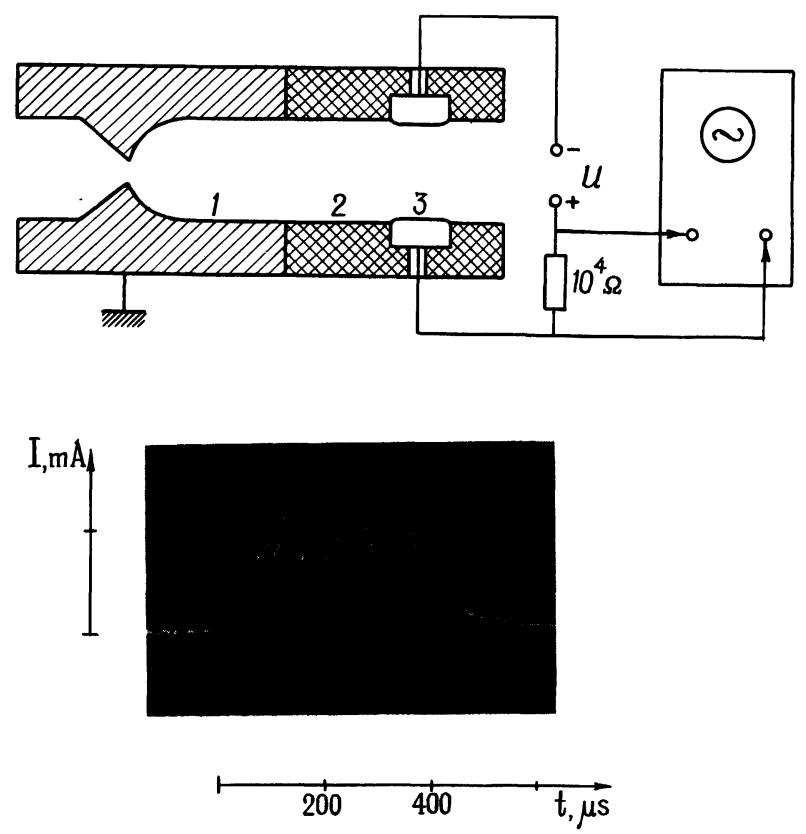

Fig. 1. - Schematic of the installation : 1, metal nozzle; 2 , dielectric constant-cross section channel; 3 , measuring electrodes and typical oscilloscope records of measuring gas conductivity.

The plasma conductivity was measured by recording the semi-self-maintained current at the gap voltage of about $100 \mathrm{~V}$. Note that the electrostatic effects have caused parasitic signals on the oscilloscope records even with no electrode voltage applied. The

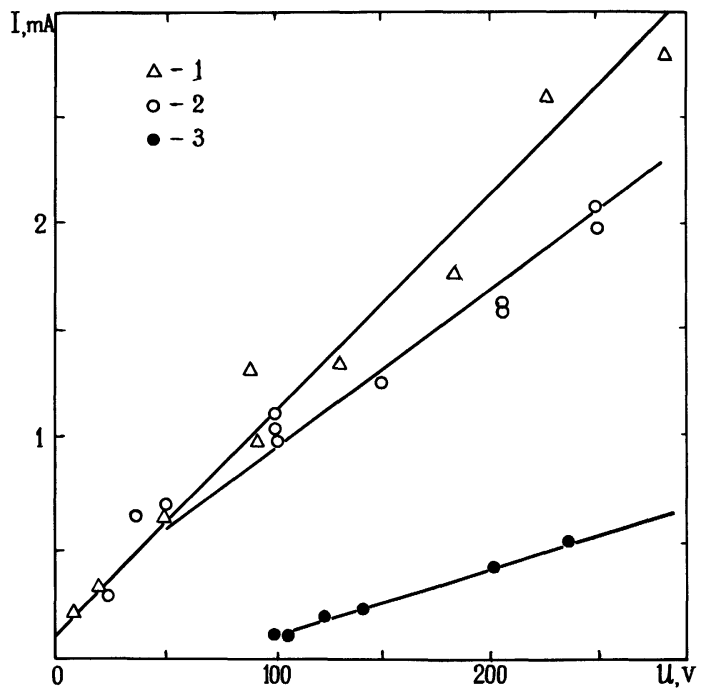

Fig. 2. - Current-voltage characteristics of measuring schemes; the stagnation temperature is $4000 \pm 150 \mathrm{~K}$ (1), $2250 \pm 100 \mathrm{~K}(2), 2800 \pm 150 \mathrm{~K}$ (3); stagnation pressure is $10 \pm 1 \mathrm{~atm}(1), 7 \pm 1 \mathrm{~atm}(2), 9 \pm 1 \mathrm{~atm}(3)$. The measuring electrodes are located at a distance of $8(1,2)$ and $14(3) \mathrm{cm}$ from the nozzle throat. The gas in the plenum chamber is $\mathrm{N}_{2}(1,2)$ and $\mathrm{CO}(3)$.

magnitude of these signals was measured and controlled on the identical measuring electrode pair and amounted to $10^{-2}-10^{-1}$ of the main measured signals. Figures 1 and 2 display typical oscilloscope records of current and current-voltage characteristics.

In the experiments, the gas flow velocity was about $2 \times 10^{5} \mathrm{~cm} / \mathrm{s}$, whereas the electron drift velocity was evaluated to be considerably higher, $2 \times 10^{6} \mathrm{~cm} / \mathrm{s}$. Since the ion drift velocity is substantially less, the electron concentration in the expanded gas flow may be evaluated as :

$$
n_{\mathrm{e}}=\frac{I / S}{e v_{\mathrm{de}}}
$$

where $S$ is the cross-section of an electrode and the value of the electron drift velocity $v_{\text {de }}(E / N)$ for nitrogen and carbon monoxide was determined in [7, 8].

It seems that $n_{\mathrm{e}}$ calculated from eq. (2) may be underestimated because of the ions space charge. On the other hand, the nonuniformity of the electric field between electrodes leads to the overestimation of the electron density. Nevertheless, there seems to be no doubt that eq. (2) would give at least the correct order of the value of $n_{\mathrm{e}}$.

Figures 2 through 6 show the values of $n_{\mathrm{e}}$ measured by the above method in adiabatically expanding $\mathrm{N}_{2}$ and $\mathrm{CO}$ flows as well as the current-voltage characteristics. As is seen in figure 2, the current-voltage characteristics of gases are in agreement with Ohm's law, which points to the fact that eq. (2) is valid under these experimental conditions. Figures $3 a$ and $3 b$ present the electron density as a function of the gas 

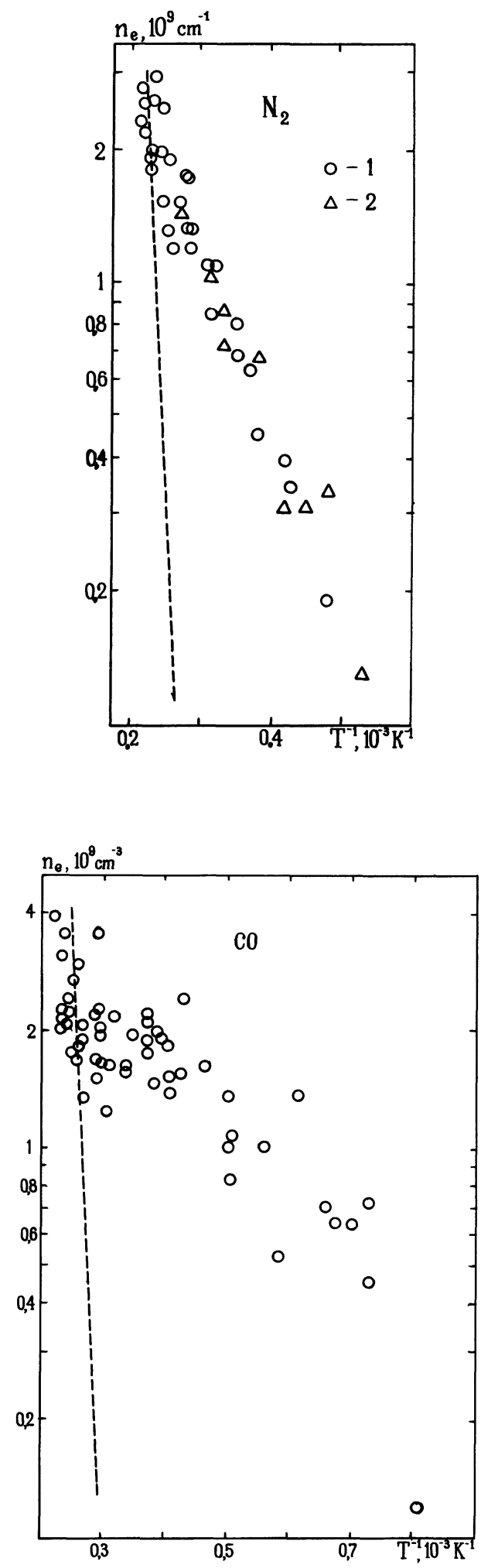

Fig. 3. - Logarithmic variation of electron concentration, $n_{\mathrm{e}}$, as a function of the inverse stagnation temperature for nitrogen $(a)$ and carbon monoxide $(b)$ at a stagnation pressure of $8.5 \pm 1 \mathrm{~atm}(a)$ and $9.5 \pm 1 \mathrm{~atm}(b)$ and at a gap voltage of $100 \mathrm{~V}$. The measuring electrodes are located at a distance of 14 (1) and $8(2) \mathrm{cm}$ from the nozzle throat. The dotted lines denote the electron density as a function of the temperature of a thermally ionized gas in the plenum chamber calculated by the Saha formula. stagnation témperature. When nitrogen served as a test gas (Fig. 3a), the experimental dependence $n_{\mathrm{e}}\left(T_{0}\right)$ is well approximated by the Arrhenius law, $n_{\mathrm{e}} \sim \exp \left(-E / T_{0}\right)$, where the effective activation ener$g y$ is about $1 \mathrm{eV}$. The flow gas temperature is $T \simeq 0.1$ $T_{0}$, i.e. considering the ionization process at this temperature evaluates the effective activation energy as $E_{\mathrm{a}} \simeq 0.1 \mathrm{eV}$. The latter is in good agreement with the results of [2] where the relation

$$
k \sim \exp (-0.1 \mathrm{eV} / T),
$$

was obtained for the rate constant of the associative ionization (1) based on a gas temperature, $T$, for nitrogen. Figure $3 a$ gives the comparison of the experimentally measured $n_{\mathrm{e}}$ with those calculated by the Saha formula for a gas temperature corresponding to the stagnation one and the density equal to the gas flow one. The coincidence of the experimental data and the Saha equilibrium calculations at gas temperatures $T_{0}>4 \times 10^{3} \mathrm{~K}$ witnesses a negligibly small contribution of the electron losses in the whole gasdynamic channel, which agrees with the data of $[9,10]$.

The analysis of a temperature dependence $n_{\mathrm{e}}\left(T_{0}\right)$ for carbon monoxide (Fig. 3b) shows that, unlike nitrogen, the pronounced exponential law is not observed in this case and at $T_{0}>2000 \mathrm{~K}$ the electron concentrations reach saturation; at $T_{0}>4000 \mathrm{~K}$ this law is governed by the exponential dependence consistent with the Saha equation. As is seen in figure $3 b$, the electron concentrations at $T_{0}>4000 \mathrm{~K}$ are below the equilibrium ones, which points to electron losses in the course of expansion.

3. Theory and discussion. - The vibrational energy of a molecule plays an important role in associative ionization processes. For instance, the reaction (1), with nitrogen used as a test gas, may proceed only when the nitrogen molecules are populated up to the 32nd vibrational energy level. Since the population of the appropriate states is negligibly small even at a temperature of several thousands degrees, the reactions of the type (1) may be provided and studied only under substantially nonequilibrium conditions. Therefore, in the present work, the adiabatic nozzle flow expansion of a preheated shock-tube compressed gas is used to attain these conditions. The vibrational relaxation processes proceeded in the adiabatic nozzle flow expansions are detailed in a number of theoretical and experimental works (cf. Review in [11]). One of the results has shown the role of the molecule vibration anharmonicity in the course of relaxation and vibrational level energy re-distribution. The difference in the vibrational relaxation times measured behind a shock wave and in the adiabatically expanding gas flow has proved to be caused by a substantial population of the upper vibrational energy levels due to specific $V-V$ transfer processes under significant separation of the vibrational and translational temperatures, $T_{\mathrm{v}}$ and $T$, in the nozzle expansion expe- 
riments. For the molecules modelled by the anharmonic oscillator this population is possible due to the difference in the energies of the vibrational quanta of the molecules participating in $V-V$ transfer processes. If in direct processes the energy is released equal to the quantum energy difference, then in inverse processes it must be compensated by the translational one. With the condition $T_{v} \gg T$ satisfied, the rates of the appropriate processes differ by the factor $\exp \left(\frac{\Delta E}{T}\right)$ where $\Delta E$ is the anharmonicity energy. As a result, the nonequilibrium vibrational distribution is formed that significantly differs from the Boltzmann distribution.

The theory developed in [12] may be adopted to find the vibrational molecule distribution, $f(v)$, under the nozzle expansion conditions. According to this theory, in the kinetics equations a set of the quantum vibrational numbers, $v$, is replaced by a continuous variable. In this case, the system of the kinetics equations for the evolution of $f(v)$ is substituted for a one-differential equation which is similar to the Fokker-Planck one. The analysis of this equation for the pulse excitation of molecule vibrations with the energy $\varepsilon_{0}$ has shown that for the upper vibrational levels, $f(v)$ is of the form given in [12] :

$$
f(v)=\frac{\left(3 / 4 \varepsilon_{0}\right)^{1 / 4}}{\sqrt{(v+1)} \tau^{3 / 4}}-\frac{1}{2 \tau} ; \quad f>0
$$

where $\tau=\int_{0}^{t} v \mathrm{~d} t$ and $v$ is the effective frequency of the $V-V$ transfer. As the frozen gas flow under the nozzle expansion, when the vibrational energy is not thermalized, is similar to the pulse excitation of the lower vibrational states, the solution of (3) will be also valid for the gas expansion conditions. In this case, $\varepsilon_{0}$ will stand for the frozen vibrational energy that can be found from :

$$
\left.u \frac{\partial \varepsilon}{\partial x}\right|_{x^{*}}=\frac{\varepsilon\left(x^{*}\right)}{\tau_{V-T}\left(x^{*}\right)}
$$

where $\varepsilon(x)$ and $\tau_{V-T}(x)$ are the equilibrium vibrational energy and $V-T$ relaxation time, respectively, for the gas parameters in the test nozzle cross section $x ; u$ is the flow velocity; $x^{*}$ is the freezing cross section coordinate. For the flow parameters typical of GDLs, the coordinate $x^{*}$ practically coincides with the nozzle throat position. The value of $\tau$ in eq. (3) for the effective number of the $V-V$ transfer processes under the nazzle expansion depends on the supersonic nozzle geometry and it is determined by $\tau=\int_{x^{*}}^{x}(v / u) \mathrm{d} x$. As follows from eq. (3), for the population of a vibrational state with the number $v$, the value of $\tau$ should exceed $\tau=(v+1)^{2} / 12 \varepsilon_{0}$, and then considerable upper vibrational level populations may be created by choosing proper nozzle parameters. Thus, for $\mathrm{N}_{2}$ flowing in the nozzle at $P_{0}=10 \mathrm{~atm}, T_{0}=2500 \mathrm{~K}$ and the expansion area ratio corresponding to a flow Mach number of $M=6, f(32) \simeq 10^{-4}$ may be attained in accordance with eq. (3). It should be noted that the energy evolution effects of highly ionized vibrational levels at constant stored vibrational energy were considered in [13] where the studies were made of the inversion properties of molecular crystals under excitation conditions. Equation (3) allows determination of the vibrational distribution function in the absence of any excitation sources, which is typical of the adiabatic nozzle expansions.

A knowledge of $f(v)$ allows determination of the electron concentration rates in the gas flow due to the associative ionization mechanism (1) :

$$
q_{v-i}=N^{2}\left[\int_{v_{0}}^{v^{* *}} f(v) \mathrm{d} v\right]^{2} K
$$

where $N$ is the gas density; $K$ the constant of the process rate $(1) ; v_{0}$ is determined by $E\left(v_{0}\right)=E_{i} / 2$ and $v^{* *}$ is the vibrational quantum number, at which the rates of the $V-V$ and $V-T$ processes are equal. The value of $K$ was evaluated in [2] by analysing the data in the case of a glow discharge in nitrogen. It was found that $K<3.5 \exp \left(-\frac{1160}{T}\right) \times 10^{-15}, \mathrm{~cm}^{3} / \mathrm{s}$. Assuming $\left(v^{* *}-v_{0}\right) \simeq 10$ and allowing for this flow rate constant, it is easy to obtain from eq. (5) that $q_{v i} \simeq 10^{12} \mathrm{~cm}^{-3} \mathrm{~s}^{-1}$.

The electron concentrations are determined by the simultaneous action of source (5) and sink due to the recombination and attachment. With these processes taken into account, the equation for the gas ionization degree $\alpha$ may be given as :

$$
u \frac{\mathrm{d} \alpha}{\mathrm{d} x}=q_{v-i} N^{-1}-\gamma \alpha-\beta \alpha^{2} N
$$

where $\gamma$ and $\beta$ are the coefficients for the attachment and volumetric recombination, respectively.

The values of $\gamma$ and $\beta$ in eq. (6) are strongly affected by the gas impurities and depend on the kind of the ions appearing in reaction (1). So, under the adiabatic nitrogen expansion the constants $\beta$ for $\mathrm{N}_{2}^{+}$and $\mathrm{N}_{4}^{+}$ ions differ by the order of magnitude and the electronegative impurities $\left(\mathrm{O}_{2}, \mathrm{CN}, \mathrm{Fe}(\mathrm{CO})_{5}\right)$ available in a gas may, by several orders, change the value of the coefficient $\gamma$. Therefore, equation (6) must be analysed with allowance for the specific experimental conditions. With the pure nitrogen flow from the nozzle, as the simple estimates show, all mechanisms of electron losses for a characteristic time of about $10^{-4} \mathrm{~s}$ may be neglected. In this case, equation (6) easily gives :

$$
\alpha=\int_{x^{*}}^{x} \frac{q_{v-i} N^{-1}}{u} \mathrm{~d} x .
$$

The evaluation of the above gas flow parameters using eq. (7) yields $\alpha \simeq 10^{-9}, n_{\mathrm{e}} \simeq 10^{8} \mathrm{~cm}^{-3}$, which 


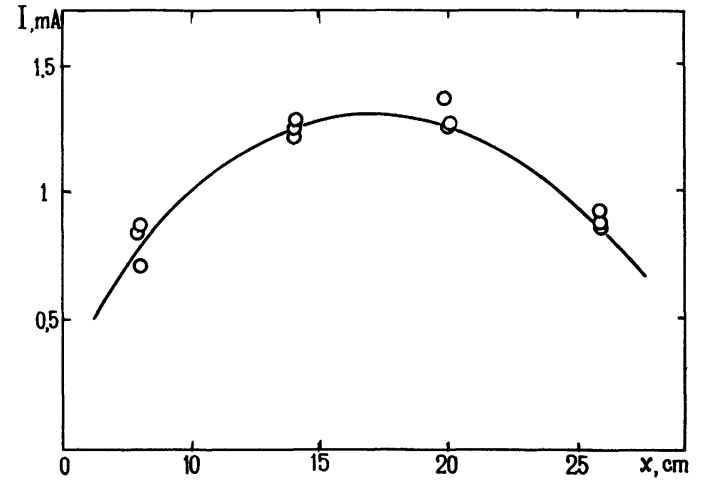

Fig. 4. - Semi-self-maintained current for the $\mathrm{CO}$ flow as a function of the distance from the nozzle throat at a gap voltage of $100 \mathrm{~V}$. The stagnation temperature is $2800 \pm 150 \mathrm{~K}$ and the stagnation pressure is $10 \pm 1 \mathrm{~atm}$.

is many orders of magnitude higher than the equilibrium values of the flow parameters even in the plenum chamber. Since at present there are no suitable methods for measuring electron concentrations, with sufficient accuracy, at a level of about $10^{8} \mathrm{~cm}^{-3}$, the associative ionization effects can be experimentally checked employing a simple estimation of $n_{\mathrm{e}}$ from the data on gas conductivity in the constant electric field, $E_{0}$. The value of $E_{0}$ is limited by a demand for the absence of the impact ionization in the gas flow. This demand is fulfilled when the value of the parameter, $E_{0} / N$, for the electron energy is less than $10^{-15} \mathrm{~V} \mathrm{~cm}{ }^{2}$, which yields $E_{0}<100 \mathrm{~V} / \mathrm{cm}$ for the above nitrogen flow parameters. Experiments were made just adopting these parameters for $E_{0} / N$.

Finally, one must consider possible mechanisms of electron losses in the $\mathrm{CO}$ flow. The presence of exponentially increasing $n_{\mathrm{e}}\left(T_{0}\right)$ on the curve section witnesses that the main mechanism of electron losses is connected with the attachment because otherwise the volumetric recombination would give a weak dependence of $n_{\mathrm{e}}$ on $T_{0}$. The analysis of the function $n_{\mathrm{e}}(x)$ (Fig. 4) confirms that the attachment processes are predominant in the $\mathrm{CO}$ flow. As the value of $n_{\mathrm{e}}$ falls approximately as much as a factor two at a distance of 17 to $24 \mathrm{~cm}$ downstream and assuming that the volumetric recombination is the main mechanism of electron losses, the condition $\beta>2 \times 10^{-5} \mathrm{~cm}^{3} \mathrm{~s}^{-1}$ would be satisfied for $\beta$, which substantially exceeds the known values of $\beta$ for $\mathrm{CO}^{+}$and $\mathrm{C}_{2} \mathrm{O}_{2}^{+}$ions. In [14], it has been emphasized that even a small amount of $\mathrm{Fe}\left(\mathrm{CO}_{5}\right)$ usually available in $\mathrm{CO}$ when stored in metal vessels and having $\gamma \simeq 10^{-9} \mathrm{~cm}^{3} \mathrm{~s}$ may favour a strong electron attachment. Assuming that in our case the electron losses are caused by this mechanism the estimation of $\eta>10^{-4}$ may be obtained for $\mathrm{Fe}(\mathrm{CO})_{5}$ concentration.

Evaluation of the vibrational level populations with $v_{0} \simeq 29$ using eq. (3) in $\mathrm{CO}$ gives negative values of $f\left(v_{0}\right)$. This points that the rates of the $V-V$ processes are small, and the excitation wave governed

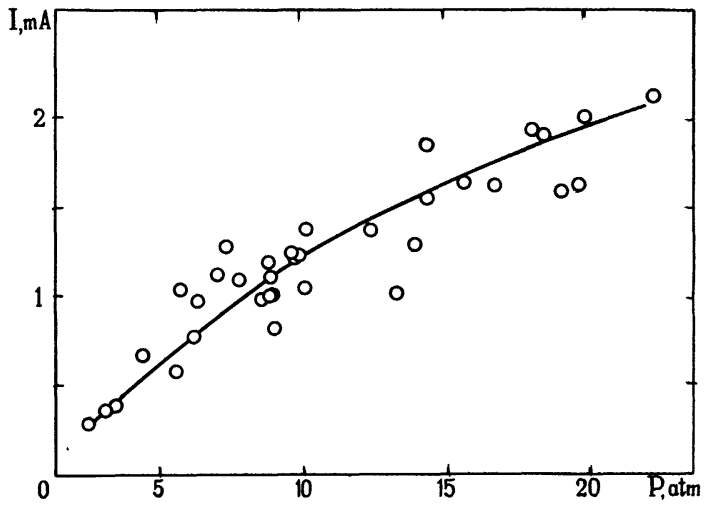

Fig. 5. - Measured current in the CO flow as a function of the stagnation pressure at a temperature of $2600 \pm 150 \mathrm{~K}$ in the plenum chamber and at a gap voltage of $100 \mathrm{~V}$. The measuring electrodes are located at a distance of $14 \mathrm{~cm}$ from the nozzle throat.

by eq. (3) cannot achieve the vibrational energy levels with $v \simeq v_{0}$. However, the experimentally observed electron concentrations occur due to a nonequilibrium ionization process.

This difference can be attributed to the approximate nature of the analytical theory [12], to an inexact choice of the $V-V$ transfer constants or the other ionization sources existing in the flow and connected with the vibrationally excited $\mathrm{CO}$ molecules (e.g. when the foreign impurities are present in the flow). In some experimental runs a small amount of molecular hydrogen, that favours a strong $\mathrm{CO}$ molecule $V-T$ relaxation, was injected into the supersonic $C O$ flow with a low translational temperature (see Fig. 6). This practically cancelled the charged particles concentration in the flow, which points out the predominant role of the vibrational $\mathrm{CO}$ molecule excitation in ionization processes.

The electron concentrations were also measured using the same parameters in $\mathrm{Ar}, \mathrm{Xe}, \mathrm{O}_{2}$ flows to check the measuring schemes and to evaluate the thermal ionization effects. No electric current was recorded in any of these gases within the framework of the sensitivity of the method and cannot be indicative

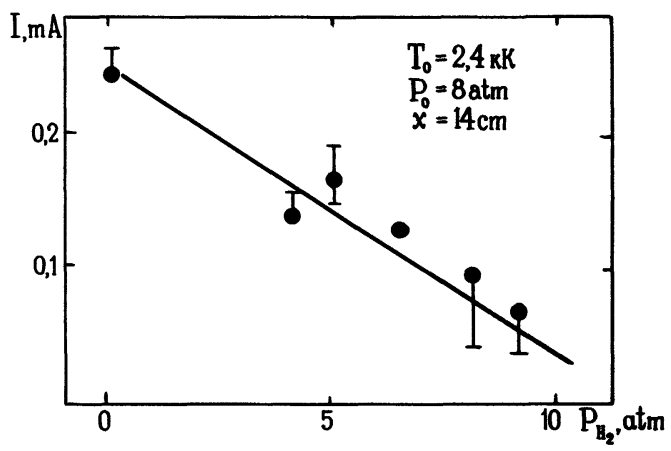

Fig. 6. - Measured current in the CO flow as a function of the pressure of hydrogen injected into the supersonic section of the nozzle. 
of electron concentrations at a level of $10^{7} \mathrm{~cm}^{-3}$ and above. This fact emphasizes that the anomaloushigh conductivity of $\mathrm{N}_{2}$ and $\mathrm{CO}$ is indeed observed in the supersonic adiabatically expanding nozzle flows.

4. Conclusion. - The observed effects of high nonequilibrium electron concentration and plasma generation in the thermally excited and vibrationally frozen molecular gas states can be widely utilized in numerous applications of molecular and laser physics, namely, for an active medium pre-ionization in an electric discharge flowing laser, in the electric subexcitation effects in the gasdynamic and chemical lasers, to serve as a diagnostic tool in evaluations of the GDL energy losses due to energy re-distribution in the upper vibrational levels, to develop electron and molecular beams using gasdynamic methods, etc. Moreover, the use and allowance for this kind of the ionization mechanisms in a relaxing gas flow may be predominant to describe the phenomena in many hypersonic gasdynamics and plasma chemistry processes when anomalously high electron concentrations appear in the gas flow past vehicles moving at cosmic velocities. The effect of electrons, ions and highly vibrationally excited molecules on the mechanisms of chemical and plasma chemical reactions can be well pronounced, too.

Acknowledgments. - The authors wish to acknowledge many helpful discussions with Drs. A. P. Napartovich and A. N. Starostin. Special appreciation is expressed to V. N. Karnyushin and A. M. Orishich for their valuable comments.

\section{References}

[1] Basov, N. G. et al, Sov. JETP 64 (1973) 108.

[2] Polak, L. S. Sergeev, I. A., Slovetsky, D. I., Teplofiz. Vysok. Temp. 15 (1977) 15.

[3] McDAniel, E. M., Collision Phenomena in Ionized Gases (John Wiley \& Sons, Inc., New YorkLondon-Sydney), 1964.

[4] Achasov, O. V. et al., Dokl. AN SSSR 253 (1980) 1373.

[5] Croshko, V. N., Soloukhin, R. I., Fomin, N. A., Fiz. Goreniya Vzryva 10 (1974) 473.

[6] Fomin, N. A., Soloukhin, R. I., Revue Phys. Appl. 14 (1979) 421.

[7] Karlov, N. V. et al., Preprint No. 91, P. N. Lebedev Physics Inst., Moscow, 1976.
[8] Konev, YU. B. et al., Reprint No. 2810, I. V. Kurchatov Atomic Power Inst., Moscow, 1976.

[9] Biryukov, A. S., Marchenko, V. M., Shelepin, L. A., Trudy, P. N., Lebedev Physics Inst. 83 (1975) 87.

[10] Davletchin, I. I., Marchenko, V. N., Sov. J. Quant. Electr. 2 (1975) 672.

[11] Gordiets, B. F., OsIPOV, A. I., ShelePIN, L. A., Kinetic Processes in Gases and Molecular Lasers (Izd. Nauka, Moscow) 1980.

[12] Zhdanok, S. A., Napartovich, A. P., Starostin, A. N., Sov. JETP 76 (1979) 130.

[13] Zeldovich, Ya. B., Ovchinnikov, A. A., Sov. JETPPis'ma 13 (1971) 636.

[14] LeE, T. G., J. Phys. Chem. 67 (1963) 360. 